\title{
A MODEL OF LOCALIZED AND NONLOCALIZED ADSORPTION FOR THE SYSTEM OF WATER AND METHANOL IN A-TYPE ZEOLITE
}

\author{
TAKuO SHIGETOMI, TOMOSHIGE NITTA \\ AND TAKASHI KATAYAMA \\ Department of Chemical Engineering, \\ Faculty of Engineering Science, \\ Osaka University, Toyonaka, Osaka 560
}

\begin{abstract}
An adsorption model combining localized and nonlocalized adsorptions is presented to interpret the adsorption isotherms of water and methanol in zeolitic sorbents of type A. The Langmuir equation is used for the localized adsorption of water and the van der Waals-type equation is suggested for nonlocalized adsorption. For methanol adsorption the extended form of the Langmuir equation based upon the model in which one molecule occupies two adjacent sites, plays a significant role. Single-component adsorptions of water and methanol on Molecular Sieve 4A are represented fairly well by means of two adjustable parameters, an adsorption equilibrium constant and a physical interaction parameter. The theory can predict the selective adsorption of water from aqueous methanol solutions without any adjustable parameters.
\end{abstract}

\section{Introduction}

The adsorption equilibrium of water in zeolitic sorbents is one of the fundamental properties for dehydration of gases and liquids and for activation of zeolites. The rational understanding of adsorption isotherms of water, however, has been beyond reach since no theoretical equations correlate the experimental data.

Statistical thermodynamics gives a sound basis for the interpretation of adsorption equilibrium. The fundamental framework for analysis of adsorption in zeolite crystals was presented by Bakajev ${ }^{1)}$ and a simplified formulation was suggested by Ruthven ${ }^{14)}$. According to Ruthven, the theory is based upon the model in which each molecule in a cavity moves independently in an averaged uniform potential field generated by the sorbate-crystal and the sorbatesorbate interactions. His final expression of the configuration integral, however, neglects the pair-wise character of the sorbate-sorbate interactions.

The basic idea developed in the present work consists of the recognition of two different types of molecules sorbed in the large $\alpha$-cage in the zeolite crystal: the localized and the nonlocalized molecules. The behavior of the localized particles attached to the surface is expressed by the Langmuir-type equation, the assumption of which is characterized by homogeneous

Received October 26, 1981. Correspondence concerning this article should be addressed to T. Katayama. T. Shigetomi is now with Asahi Chemical Industry Co., Ltd., Nobeoka 882. and independent active sites. A simplified expression, based upon the free volume theory, is suggested in the first section for nonlocalized molecules confined in a space surrounded by localized molecules.

The above adsorption model is based on the relative size of water molecule to the diameter of $\alpha$ cage. The theory is extended and applied to methanol adsorption and to coadsorption from liquid mixtures of water and methanol on Molecular Sieve 4A, experimental data for which have recently been given by the authors $^{12)}$.

\section{Adsorption Model and the Partition Function}

\section{1 Model description}

Let $M$ be the number of sites on the inner surface of one unit $\alpha$-cage. All sites are assumed to be homogeneous and independent. The number of molecules adsorbed on the sites is denoted by $N\left(N \leq N^{0}\right)$. The molecules which enter into the cage after all the sites have been occupied $\left(N=N^{0}\right)$ are assumed to move about in the remaining space. Therefore, their behavior may be expressed by the van der Waals-type (nonlocalized) equation. The number of these fluidlike molecules is denoted by $s$.

Figure 1 shows the schematic adsorption model in the $\alpha$-cage where $\varepsilon_{\mathrm{ad}}$ is the interaction energy between an adsorbed molecule and the surface. The symbol $\phi$ is the interaction energy between any free molecule and the adjacent attaching molecules and $\phi$ is that between any free molecule and an adjacent free molecule. 


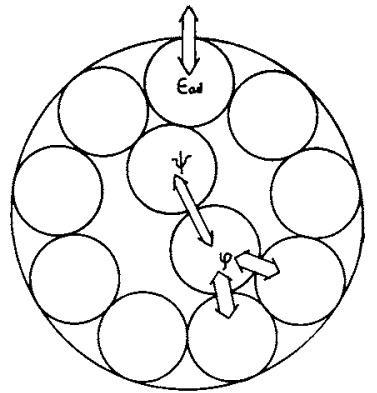

Fig. 1 Adsorption model in $\alpha$-cage

\subsection{Expression of the partition function}

The partition function for the system of $N$ localized adsorbate and $M$ independent sites is given as

$$
Q(N)=g(N, M) \exp \left(-\frac{N \varepsilon_{\mathrm{ad}}}{k T}\right) \quad\left(N \leq N^{0}\right)
$$

where $g(N, M)$ is the number of distinguishable ways of distributing the adsorbed molecules over the sites $^{4,7)}$. The partition function of the van der Waals fluid composed of $s$ molecules is given as?

$$
\frac{1}{s !}\left[\frac{V_{f}}{A^{3}} \exp \left(\frac{a_{\mathrm{v}} \rho}{k T}\right)\right]^{s}
$$

where $V_{\mathrm{f}}$ is a free volume and $-a_{\mathrm{\gamma}} \rho$ is the average attractive potential shared with one molecule.

Then for the system of $N^{0}$ localized adsorbates and $s$ mobile molecules in the unit cage, the partition function may be expressed as

$$
Q\left(N^{0}+s\right)=Q\left(N^{0}\right) \frac{1}{s !}\left(\frac{V_{\mathrm{f}_{-}}}{A^{3}}\right)^{s} \exp \left(-\frac{s \Phi}{k T}\right)
$$

where $\Phi$ is the average potential field provided by the adjacent molecules attached to the wall and other free molecules.

The Carnahan-Starling equation is used for the freevolume expression as $^{2,15 y}$

$$
V_{\mathrm{f}}=V^{\prime} \exp \left\{\frac{\xi(3 \xi-4)}{(1-\xi)^{2}}\right\}
$$

where the volume available for the fluid-like molecules, $V^{\prime}$, is calculated under the assumption that each molecule attached to the surface excludes the volume that would be allocated to a molecule in the liquid state.

$$
V^{\prime}=V_{\text {cage }}-N^{0}\left(v^{l} / L\right)
$$

The reduced density $\xi$ is calculated by means of the hard-core volume of a molecule $v^{*} / L$ as

$$
\xi=s\left(v^{*} / L\right) / V^{\prime}
$$

The average potential $\Phi$ is expressed from the two contributions of $\phi$ and $\phi$ as

$$
\Phi=\phi+\frac{1}{2} \phi(s-1)
$$

The combinatorial factor $g(N, M)$ has different expressions according to the number of sites that an adsorbed molecule occupies. When one site is taken for one molecule, the expression for $g$ is given as

$$
g_{(1)}=\frac{M !}{N !(M-N) !}
$$

Equation (7) is used for water adsorption.

When two sites are covered by one adsorbed molecule, the approximate expression for $g$ of Eq. (8) is obtained; its derivation procedure is similar to that of Flory $^{3)}$, who evaluated the number of configurations of polymer solutions.

$$
g_{(2)}=\frac{M !}{N !(M-2 N) !}\left(\frac{Z}{M-1}\right)^{N}
$$

where $z$ is the coordination number of a site. The degree of approximation of Eq. (8) was checked in the case of a regular dodecahedron $(M=20)$ by comparing the exact value of $g_{(2)}$ calculated by means of a digital computer. A brief description of the method for computing the number of distinguishable ways is given in Appendix 1 along with a comparison of the exact and approximate values for $g_{(2)}$. Equation (8) is used for methanol adsorption.

The grand canonical partition function $\Xi$ is given by means of the absolute activity $\lambda(=\exp (\mu / k T))$ as

$$
\Xi=\sum_{N=0}^{N^{0}} Q(N) \lambda^{N}+\sum_{s=1} Q\left(N^{0}+s\right) \lambda^{N^{0}+s}
$$

The average number of adsorptions per unit $\alpha$-cage, $\langle N+s\rangle$, is calculated from the conventional procedure as

$$
\langle N+s\rangle=\frac{\lambda}{\Xi} \cdot \frac{\partial \Xi}{\partial \lambda}
$$

The adsorption equilibrium constant $K$, relative to the saturation vapor, is defined by Eq. (11).

$$
K=\exp \left[\left(\mu^{0}-\varepsilon_{\mathrm{ad}}\right) / k T\right]
$$

where $\mu^{0}$ is the chemical potential of pure component at saturation. The absolute activity $\lambda$ is related to the activity $\left.a\left(=\exp \left\{\mu-\mu^{0}\right) / k T\right\}=P / P^{0}\right)$ as

$$
K a=\lambda \exp \left(-\varepsilon_{\mathrm{ad}} / k T\right)
$$

and

$$
\lambda=P^{0} a \Lambda^{3} / k T
$$

The final expression for $\langle N+s\rangle$ is obtained as

$$
\begin{aligned}
\langle N+s\rangle= & \frac{1}{\Xi}\left[\sum_{N=0}^{N^{0}} N \cdot g(N, M)(K a)^{N}\right. \\
& +g\left(N^{0}, M\right)(K a)^{N^{0}} \sum_{s=1} \frac{\left(N^{0}+s\right)}{s !}\left(\frac{V_{\mathrm{f}} P^{0} a}{k T}\right)^{s} \\
& \left.\times \exp \left\{-\frac{\phi s}{k T}-\frac{\phi s(s-1)}{2 k T}\right\}\right]
\end{aligned}
$$

A numerical method was used to calculate the average adsorption capacity $\langle N+s\rangle$ through Eq. (14). When 
the number of free molecules, $s$, increases, the term $V_{\mathrm{f}}$ decreases faster than the attractive potential terms $\phi$ and $\phi$; therefore, in practice the sum over $s$ was truncated when the value of the reduced density $\xi$ became greater than 0.75. According to Gramlich and $\mathrm{Meier}^{5}$, , only water molecules are assumed to be accommodated in the small $\beta$-cage, four at maximum. The Langmuir equation is used for the adsorption, and the calculated number of water molecules is added to that in the $\alpha$-cage. The value for $K$ in the $\beta$-cage was assumed to be identical to that in the $\alpha$-cage.

\section{3 Extension to coadsorption}

Extension of the model to multicomponent adsorption is straightforward. In the case of a binary mixture of water (1) and methanol (2) the grand partition function $\Xi$ is given as

$$
\begin{aligned}
E= & \sum_{N_{1}} \sum_{N_{2}} \frac{M !}{N_{1} ! N_{2} !\left(M-N_{1}-2 N_{2}\right) !}\left(K_{1} a_{1}\right)^{N_{1}}\left(\frac{K_{2} a_{2} z}{M-1}\right)^{N_{2}} \\
& +\sum_{N_{2}{ }^{0}} \frac{M !}{N_{1}^{0} ! N_{2}^{0} !}\left(K_{1} a_{1}\right)^{N_{1}}\left(\frac{K_{2} a_{2} z}{M-1}\right)^{N_{2}{ }^{0}} \\
& \times \sum_{s_{1}} \sum_{s_{2}} \frac{1}{s_{1} ! s_{2} !}\left(\frac{V_{\mathrm{f}} P_{1}^{0} a_{1}}{k T}\right)^{s_{1}}\left(\frac{V_{\mathrm{f}} P_{2}^{0} a_{2}}{k T}\right)^{s_{2}} \\
& \times \exp \left\{-\frac{s_{1} \Phi_{1}}{k T}-\frac{s_{2} \Phi_{2}}{k T}\right\}
\end{aligned}
$$

where

$$
\begin{gathered}
\Phi_{i}=\phi_{i i} \theta_{i}+\phi_{i j} \theta_{j}+\frac{1}{2}\left\{\phi_{i i}\left(s_{i}-1\right)+\phi_{i j} s_{j}\right\} \\
\theta_{1}=N_{1}^{0} / M \\
\theta_{2}=2 N_{2}^{0} / M
\end{gathered}
$$

The free volume of binary mixture $V_{\mathrm{f}}$ in Eq. (15) is calculated by means of Eq. (3); The reduced density $\xi$ is given by Eq. (19).

$$
\xi=\left(s_{1} v_{1}^{*}+s_{2} v_{2}^{*}\right) / L V^{\prime}
$$

The average number of adsorptions for component $i$, $\left\langle N_{i}+s_{i}\right\rangle$, is given as

$$
\left\langle N_{i}+s_{i}\right\rangle=\frac{a_{i}}{\Xi} \cdot \frac{\partial \Xi}{\partial a_{i}}
$$

\section{Application to Synthetic Zeolite NaA}

\subsection{Assigned parameters}

There are many parameters to be assigned when the model equation is applied to the systems of water and/or methanol in synthetic zeolite NaA. Among the parameters, $M, z$, and $V_{\text {cage }}$ are related to the cavity structu:e; the values of 20 for $M$ and 3 for $z$ were fixed after several values had been examined. The diameter of $\alpha$-cage $\left(1.14 \mathrm{~nm}^{13)}\right)$ yields a volume of $0.776 \mathrm{~nm}^{3}$; however, the value of $0.733 \mathrm{~nm}^{3}$ for $V_{\text {cage }}$ was used by subtracting the volume of twelve sodium ions (the diameter of $0.19 \mathrm{~nm}$ for $\mathrm{Na}^{+6}$ ) from the sphere volume.
Table 1 Parameters used for the calculation of adsorption isotherms of water and methanol on Molecular Sieve 4A at $25^{\circ} \mathrm{C}$

\begin{tabular}{lcc}
\multicolumn{1}{c}{ Component } & Water & Methanol \\
\hline$K[-]$ & 803 & 1854 \\
$\phi / k[\mathrm{~K}]$ & -3316 & -5225 \\
$\phi / k[\mathrm{~K}]$ & -911 & -709 \\
$v^{*}\left[\mathrm{~cm}^{3} \cdot \mathrm{mol}^{-1}\right]$ & 9.456 & 19.83 \\
$v^{l}\left[\mathrm{~cm}^{3} \cdot \mathrm{mol}^{-1}\right]$ & 18.068 & 40.733 \\
$P^{0}[\mathrm{~Pa}]$ & 3159.8 & 16629 \\
\hline$M=20$ & & \\
$z=3$ & & \\
$V_{\text {eage }}=0.733 \mathrm{~nm}^{3}$ & & \\
\end{tabular}

The parameters $v^{*}$ and $\psi$ are involved solely in the adsorbate; they were determined from the two liquid properties of density and vapor pressure at each temperature. The details of equations used to fit the saturation liquid properties are given in Appendix 2.

The parameter $K$ is the direct measure of the intensity of adsorption per molecule interacting with the solid surface. The parameter $\phi$ may depend upon the number of molecules adjacent to a fluid-like molecule among the molecules attached to the surface, which may be related to the curvature of the surface and the molecular size. Since the two parameters $K$ and $\phi$ were difficult to estimate, they were determined by means of a non-linear least square method so as to give the best fit with a one-component adsorption isotherm. All values at $25^{\circ} \mathrm{C}$ used in this work are summarized in Table $\mathbf{1}$ for the system of water and methanol on Molecular Sieve 4A. The sensitivity of the parameter $K$ on an adsorption isotherm is high, as in the case of the Langmuir equation; however, the parameter $\phi$ is rather insensitive. The cross-terms of $\phi_{i j}$ and $\phi_{i j}$ were calculated from the geometric mean assumption though theoretical justification is lacking.

The mole quantity of unit cages per gram of adsorbent, which was necessary for comparing the calculated values with the experimental data, was taken as 0.453 $\mathrm{mmol} \cdot \mathrm{g}^{-1}$ for Molecular Sieve $4 \mathrm{~A}$ from the datafitting of water adsorption equilibrium at $25^{\circ} \mathrm{C}$.

\section{2 Water adsorption}

The average number of water molecules adsorbed per unit cage at $25^{\circ} \mathrm{C}$ is shown in Fig. 2 against the logarithm of the activity of water. The theoretical curve for $\alpha$-cage has two inflection points; one is the half site occupancy point associated with the conventional Langmuir-type equation and the other is due to the van der Waals behavior of fluid-like molecules. The solid line in the figure shows the water adsorption on Molecular Sieve 4A taken from Linde's technical information and converted to the units of $m m o l-\mathrm{H}_{2} \mathrm{O} /$ g-adsorbent and the activity of water.

In the region of high activity of water the adsorp- 


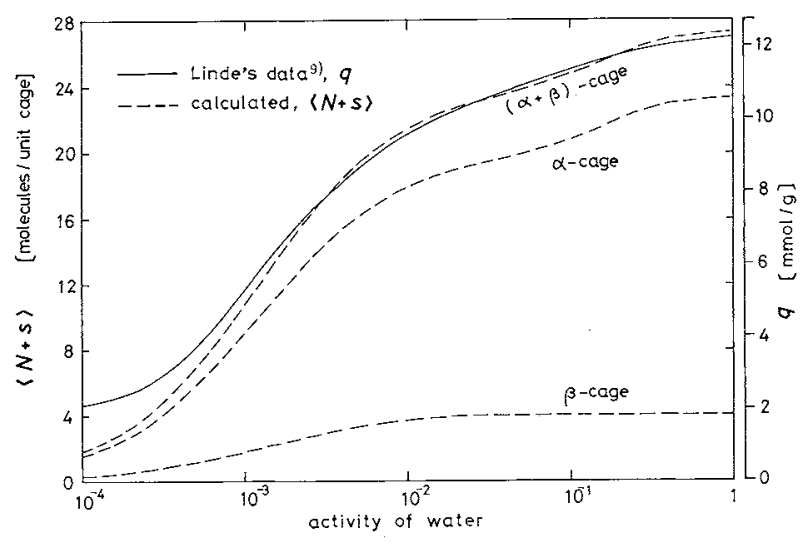

Fig. 2 Adsorption isotherm of water in Molecular Sieve $4 \mathrm{~A}$ at $25^{\circ} \mathrm{C}$

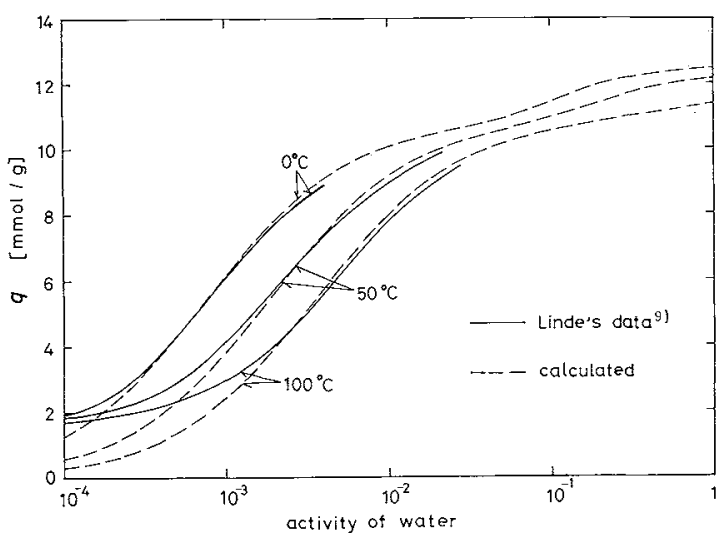

Fig. 3 Adsorption isotherms of water in Molecular Sieve $4 \mathrm{~A}$

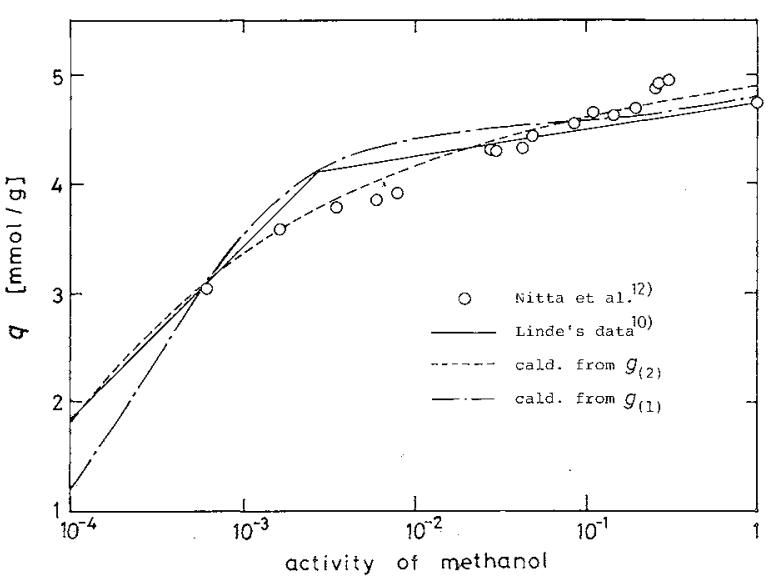

Fig. 4 Adsorption isotherm of methanol in $\mathrm{M}$ (O)lecular Sieve $4 \mathrm{~A}$ at $25^{\circ} \mathrm{C}$

tive capacity decreases gradually with decreasing activity, which may be attributed to the behavior of fluid-like molecules in the $\alpha$-cage. In the region of low activity of water the experimental curve deviates upward, which may indicate the existence of sites interacting more strongly with water molecules. The number of the most active sites is estimated to be five or six. However, it should be noted that the experimental adsorption isotherm in the region of low water content is doubtful as Morris ${ }^{11}$ has already pointed out from the calculation of the heats of sorption.

Figure 3 shows water adsorption isotherms at 0 , 50 , and $100^{\circ} \mathrm{C}$ calculated from one adjustable parameter $K$ at each temperature. The values of $\phi$ were evaluated from the assumption that

$$
(\phi / \phi)_{T}=(\phi / \phi)_{298.1 .5 \mathrm{~K}}
$$

The energy of water adsorption from liquid state, $\Delta H_{\mathrm{a}}$, was calculated as $-13.0 \mathrm{~kJ} \cdot \mathrm{mol}^{-1}$ from the temperature variation of the adsorption equilibrium constant $K$. Since the heat of vaporization of water, $\Delta H_{\mathrm{v}}$, is $44.0 \mathrm{~kJ} \cdot \mathrm{mol}^{-1}$ at $25^{\circ} \mathrm{C}$, the heat of sorption relative to water vapor is $-57.0 \mathrm{~kJ} \cdot \mathrm{mol}^{-1}$ for the adsorption process of the Langmuir type in this model.

\subsection{Methanol adsorption}

The curves for the methanol adsorption isotherm at $25^{\circ} \mathrm{C}$ are shown in Fig. 4. A solid line is taken from Linde's technical information ${ }^{10)}$ and circles are from the authors' data of liquid-phase adsorption ${ }^{12)}$. A broken line is the theroretical adsorption isotherm by use of $g_{(2)}$ expressed in Eq. (8) with $M=20$, and the one-dot chain line is that of $g_{(1)}$ in Eq. (7) with $M=10$. It is obvious that the broken line represents the data points within their experimental errors. The average number of methanol molecules adsorbed from pure liquid is calculated as 10.79 , which indicates that there remains a slightly small space for a fluid-like molecule to be accommodated.

A gradual decrease of methanol adsorption isotherm with decreasing activity is attributed mainly to the functional form of $g_{(2)}$.

\section{4 Coadsorption of water and methanol}

Experimental data for the coadsorption of water and methanol on Molecular Sieve 4A have been obtained by the authors ${ }^{12}$, who used the liquid-phase adsorption method combined with measurement of heterogeneous density of the liquid mixture and the adsorbent at equilibrium. Figure 5 shows the theoretical (solid line) and experimental (circles and squares) values for the amount of adsorption at $25^{\circ} \mathrm{C}$. The values for activity coefficients of each component, which are necessary in calculating the activity at a specified composition, were evaluated by means of the table recommended by one of the authors ${ }^{8}$. Two solid lines in the figure are essentially the predictions since the cross-term of interaction parameters $\phi_{i j}$ and $\psi_{i j}$ were calculated from the geometric mean assumption. Some other values for $\phi_{i j}$ and $\phi_{i j}$ were used to calculate the coadsorption isotherm. However, the parameters were so insensitive that the calculated results were almost the same as given in Fig. 5.

The adsorption model can predict the selective adsorption of water fairly well though the value of $K$ 
characteristic of the interaction per molecule between water and the surface is smaller than that between methanol and the surface. This result may be interpreted as showing that the real measure of selectivity is not $K_{1} / K_{2}$ but $K_{1}^{2} / K_{2}$ because two molecules of water can attach to the two sites which accommodate only one molecule of methanol.

The predicted curve for water adsorption gives larger values than the experimentals by 0.7 to $1.3 \mathrm{mmol} \cdot \mathrm{g}^{-1}$ though the prediction for methanol adsorption is good within experimental error. If the number of water molecules in the $\beta$-cage is reduced from four to two, the discrepancy between theory and experiment may diminish on the whole.

\section{Concluding Remarks}

The adsorption isotherms of water and methanol in Molecular Sieve 4A are represented fairly well by the localized and nonlocalized adsorption model presented. A gradual decrease of adsorption in the region of high water content with decreasing activity is attributed to the behavior of several fluid-like molecules in the $\alpha$-cage. In the case of methanol adsorption, the combinatorial factor that accounts for the large molecular size of methanol plays a significant role.

The adsorption model predicts the selective adsorption of water from an aqueous methanol solution. A measure of selectivity is shown not to be the ratio of the adsorption equilibrium constants $K_{1} / K_{2}$ but of $K_{1}^{2} / K_{2}$ (the suffix 1 stands for water and 2 for methanol). This result will be generalized to mixtures having different molecular sizes.

The introduction of heterogeneous energy sites will be necessary for better representation of the adsorption isotherms. However, mathematical difficulties are expected, especially for methanol adsorption. How to define the dimension of the site seems very interesting and important for the understanding of adsorption equilibrium.

\section{Appendix}

1. The number of ways of distributing molecules covering two adjacent sites over a regular dodecahedron

Twenty vertices of a regular dodecahedron are numbered from 1 to 20. Consider the case in which one molecule occupies two adjacent sites. There are thirty sides in a dodecahedron; therefore, the number of ways of distributing the first molecule is 30 if each segment of a molecule is equivalent (indistinguishable). Locate the first molecule on sites 1 and 2. Let $w(N)$ be the number of ways of distributing $N$ indistinguishable molecules having equivalent segments over twenty vertices, where $N$ includes the first molecule placed on sites 1 and 2 . Then, the number of ways of distributing $N$ indistinguishable molecules having the symmetric number $\sigma, g(N)$, is calculated as

$$
g(N)=\frac{30}{N} \cdot\left(\frac{2}{\sigma}\right)^{N} \cdot w(N)
$$

Evaluation of $w(N)$ was performed by counting the possible

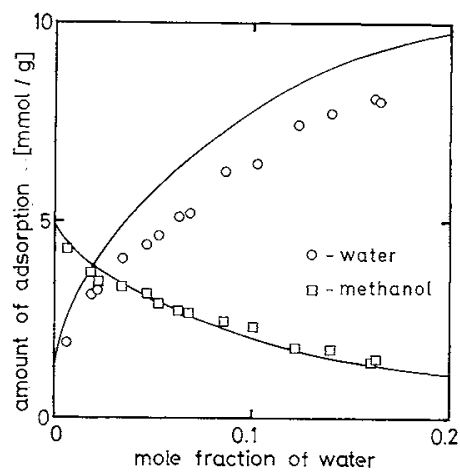

Experimental data were taken from Nitta et al. ${ }^{12)}$

Fig. 5 Coadsorption equilibria from water and methanol solutions in Molecular Sieve $4 \mathrm{~A}$ at $25^{\circ} \mathrm{C}$

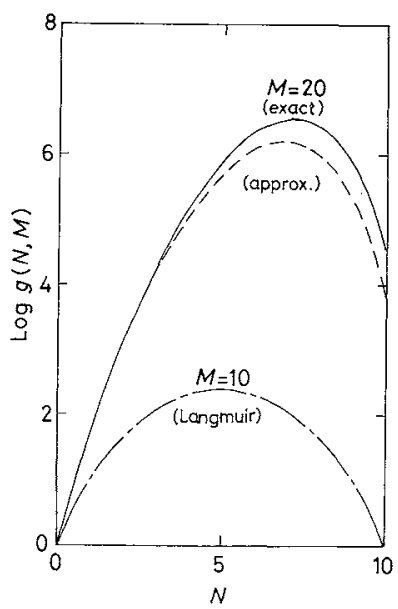

Fig. A-1 Comparison of different expressions of the combinatorial factor $g(N, M)$

Table A-1 Comparison of the combinatorial factor $g(N, 20)$ for molecules having two segments: approximate values calculated from $\mathrm{Eq}$. (8) and exact values calculated from digital computation

\begin{tabular}{rrr}
$N$ & Approximate & \multicolumn{1}{c}{ Exact } \\
\hline 0 & 1 & 1 \\
1 & 60 & 60 \\
2 & 1449.5 & 1500 \\
3 & 18309.1 & 20320 \\
4 & 131536.7 & 162480 \\
5 & 548300.5 & 783168 \\
6 & 1298606.3 & 2227520 \\
7 & 1640344.9 & 3494400 \\
8 & 971356.8 & 2626560 \\
9 & 204475.1 & 716800 \\
10 & 6457.1 & 36864 \\
\hline
\end{tabular}

configurations of the numbered molecules such that largernumber molecules occupy large-number sites and that the second segment of each molecule must occupy a site of larger number than that of the first segment. Computation was performed by means of a microdigital computer in an hour.

The exact and approximate values for the combinatorial factor $g(N, M)$ in the case where $M=20$ and $\sigma=1$ are compared in Table A-1 and in Fig. A-1. The asymmetric shape in In $g(N, 20)$ is approximately expressed by Eq. (8) in the text; however, Eq. (7) gives a symmetrical curve if the number of 
sites $M$ is assumed to be ten.

2. Evaluation of hard-core volume $v^{*}$ and pair interaction parameter $\phi$

The partition function of the van der Waals-type fluid with the Carnahan-Starling equation for a repulsive interaction term is expressed $\left.a^{2}, 7,15\right\rangle$

$$
Q(s, V)=\frac{1}{s !}\left(\frac{V}{A^{3}}\right)^{s} \exp \left\{\frac{s \xi(3 \xi-4)}{(1-\xi)^{2}}+\frac{a_{v} s^{2}}{V k T}\right\}
$$

where

$$
\xi=s\left(v^{*} / L\right) / V
$$

and $a_{\mathrm{v}}$ is a constant related to the attractive interaction. The equation of state derived from Eq. (A-2) is

$$
\frac{P^{0} v^{l}}{R T}=1+\frac{\xi(4-2 \xi)}{(1-\hat{\xi})^{3}}-\frac{a_{\mathrm{v}}}{\left(v^{i} / L\right)} \overline{k T}
$$

where $v^{l}$ is the molar volume of a liquid at saturation pressure $P^{0}$. By equating the chemical potentials of liquid and vapor phases, another equation concerning $P^{0}$ and $v^{l}$ is obtained as

$$
\ln \frac{P^{0} v^{l}}{R T}=\frac{\xi\left(3 \xi^{2}-9 \xi+8\right)}{(1-\xi)^{3}}-\frac{2 a_{\mathrm{v}}}{\left(v^{l} / L\right) k T}
$$

The simultaneous equations (A-4) and (A-5) for $v^{*}$ and $a_{v}$ are solved numerically by an iterative procedure. Although $2 a_{\mathrm{v}}(s / V)$ is the average potential energy of interaction between any one molecule and all others in liquid state, it is assumed to be the sum of pair-interaction energies with the nearest neighbors, $z^{l} \psi$, where $z^{l}$ is the coordination number in liquid state. The intermolecular energy for each pair of molecules with the nearest neighbor, $\phi$, is then calculated as

$$
\phi=\frac{2}{z^{l}} \cdot \frac{a_{\nabla}}{\left(v^{l} / L\right)}
$$

The value of ten is assumed for $z^{l}$.

\section{Nomenclature}

$a \quad=$ activity

$a_{\vee} \quad=$ van der Waals constant for attraction force

$g \quad=$ combinatorial factor

$H \quad=$ enthalpy

$K=$ adsorption equilibrium constant

$k \quad=$ Boltzmann constant

$L \quad=$ Avogadro constant

$M \quad=$ number of sites in a unit cage

$N \quad=$ number of molecules adsorbed on a surface

$N^{0} \quad=$ maximum number of molecules adsorbed on a surface

$=$ pressure

$=$ saturation vapor pressure

$=$ canonical partition function

$=$ amount of adsorption

= gas constant $[\mathrm{mmol} / \mathrm{g}]$

$=$ number of nonlocalized molecules

$\left[\mathrm{J} \cdot \mathrm{K}^{-1} \cdot \mathrm{mol}^{-1}\right]$

$=$ absolute temperature

$V=$ volume
$\begin{array}{ll}V_{\text {cage }} & =\text { volume of a cage } \\ V_{\mathrm{f}} & =\text { free volume }\end{array}$

$\left[\mathrm{m}^{3}\right]$

$V_{\mathrm{f}} \quad=$ free volume

$V^{\prime} \quad=$ volume in which nonlocalized molecules move about

$v \quad=$ molar volume

$v^{*} \quad=$ hard core volume

$w(N) \quad=$ number of ways of distributing $N$ indistinguishable molecules having equivalent segments over sites

$\left[\mathrm{m}^{3}\right]$

$\left[\mathrm{m}^{3} \cdot \mathrm{mol}^{-1}\right]$ $\left[\mathrm{m}^{8} \cdot \mathrm{mol}^{-1}\right]$

$z \quad=$ coordination number

$z^{l} \quad=$ coordination number of liquid

$[-]$

$[-]$

$[-]$

$\varepsilon_{\mathrm{ad}} \quad=$ adsorption free energy per molecule $\quad[\mathrm{J}]$

$\theta \quad=$ fraction of surface coverage $[-]$

$A=$ de Brogli thermal wave length [m]

$\lambda \quad=$ absolute activity $[-]$

$\mu \quad=$ chemical potential $\quad[\mathrm{J}]$

$\mu^{0} \quad=$ chemical potential of pure liquid

$E \quad=$ grand canonical partition function $\quad[-]$

$\xi \quad=$ reduced density $[-]$

$\sigma \quad=$ symmetric number $\quad[-]$

$\Phi=$ potential energy per fluid-like molecule [J]

$\phi \quad=$ potential energy between a free molecule and the adjacent molecules attached to active sites

$\phi \quad=$ pair potential energy of fluid-like molecules

$\langle$ Subscripts〉

$$
\begin{array}{ll}
1 & =\text { water } \\
2 & =\text { methanol } \\
i j & =\text { refers to the pair of } i \text { and } j \text { components }
\end{array}
$$

\section{Literature Cited}

1) Bakajev, V. A.: Dokl. Akad. Nauk. USSR, 167, 369 (1966).

2) Carnahan, N. F. and K. E. Starling: AIChE J., 18, 1184 (1972).

3) Flory, P. J.: J. Chem. Phys., 12, 425 (1944).

4) Fowler, R. H. and E. A. Guggenheim: "Statistical Thermodynamics", Cambridge Univ. Press (1965).

5) Gramlich, V. and W. M. Meier: Zeit. Krist., 133, 134 (1971).

6) Heslop, R. B. and K. Jones: "Inorganic Chemistry", Elsever (1976), translated into Japanese by Y. Saito, Tokyo Kagaku Dojin (1977).

7) Hill, T. L.: "An Introduction to Statistical Thermodynamics", Addison-Wesley, Massachusetts (1960).

8) Katayama, T.: Kagaku Kögaku, 26, 490 (1962).

9) Linde's technical information, Form 9690-E.

10) Linde's technical information, Form XF-20.

11) Morris, B.: J. Colloid and Interface Science, 28, 149 (1968).

12) Nitta, T., K. Kitaoh, T. Shigetomi, and T. Katayama: Kagaku Kogaku Ronbunshu, 8, 334 (1982).

13) Reed, T. B. and D.W. Breck: J. Am. Chem. Soc., 78, 5972 (1956)

[K] 14) Ruthven, D. M.: Nature, Phys. Sci., 232, 70 (1971).

$\left.\left[\mathrm{m}^{3}\right] \quad 15\right)$ Vera, J. H. and J. M. Prausnitz: Chem. Eng. J., 3, 1 (1972). 